\title{
Fascia iliaca block prophylaxis for hip fracture patients at risk for delirium: a randomized placebo-controlled study
}

\author{
George Mouzopoulos · George Vasiliadis • \\ Nikolaos Lasanianos · George Nikolaras · \\ Emanouil Morakis $\cdot$ Michalis Kaminaris
}

Received: 2 June 2009/Accepted: 24 July 2009/Published online: 19 August 2009

(C) Springer-Verlag 2009

\begin{abstract}
Background Recent studies have indicated that unmanaged pain, both acute and chronic, can affect mental status and might precipitate delirium, especially in elderly patients with hip fractures. The aim of this study was to assess the effectiveness of fascia iliaca compartment block (FICB) for prevention of perioperative delirium in hip surgery patients who were at intermediate or high risk for this complication.

Materials and methods On admission, all included patients were divided into three groups according to low, intermediate or high risk for perioperative delirium. Eligible patients (those classified as at intermediate or high risk for developing delirium) were sequentially randomly assigned to study treatment (FICB prophylaxis or placebo) according to a computer-generated randomization code.
\end{abstract}

\footnotetext{
G. Mouzopoulos - N. Lasanianos - G. Nikolaras - E. Morakis · M. Kaminaris

Orthopaedic Department, Evangelismos Hospital,

Athens, Greece

G. Vasiliadis

Euromedica Geniki Kliniki of Thessaloniki,

Thessaloniki, Greece

G. Vasiliadis

3rd Department of Neurology, Aristotle's University

of Thessaloniki, Thessaloniki, Greece

G. Mouzopoulos

Post-Graduate Department of Biostatistics, Medical School, University of Athens, Athens, Greece

G. Mouzopoulos $(\bowtie)$

Sof. Venizelou 23, Peristeri Attikis, 12131 Athens, Greece

e-mail: gmouzopoulos@yahoo.gr
}

The primary outcome was perioperative delirium. Diagnosis of the syndrome was defined using the Diagnostic and Statistical Manual of Mental Disorders, 4th edition (DSM-IV) and Confusion Assessment Method (CAM) criteria. Secondary outcome variables were severity of delirium and delirium duration.

Results Delirium occurred in 33 (15.94\%) out of 207 patients randomized to FICB prophylaxis or the placebo group. Incidence of delirium in the FICB prophylaxis group was $10.78 \%$ (11/102), significantly different from the incidence $(23.8 \%, 25 / 105)$ in the placebo group [relative risk 0.45 , 95\% confidence interval (CI) $0.23-0.87$ ]. Nine of 17 patients with high risk for delirium and included in the FICB prophylaxis group developed delirium, whereas 10 of 16 high-risk patients included in the placebo group became delirious (relative risk 0.84, CI 0.47-1.52). Two of 85 patients with intermediate risk for delirium and included in the FICB prophylaxis group developed delirium, whereas 15 of 89 intermediate-risk patients included in the placebo group became delirious (relative risk 0.13, CI 0.03-0.53). Severity of delirium according to the highest value of the DRSR-98 during an episode with delirium in patients in the FICB prophylaxis group was on average 14.34, versus 18.61 in the placebo group (mean difference $4.27,95 \%$ CI $1.8-5.64, P<0.001)$. Mean duration of delirium in the FICB prophylaxis group was significantly shorter than in the placebo group (FICB 5.22 days versus placebo 10.97 days, $95 \%$ CI 3.87-7.62, $P<0.001)$.

Conclusion No significant difference was found among high-risk patients between FICB prophylaxis and placebo groups in terms of delirium incidence. However, FICB prophylaxis significantly prevented delirium occurrence in intermediate-risk patients. Thus FICB prophylaxis could be beneficial, particularly for intermediate-risk patients. 
Keywords Hip fracture - Delirium · Pain .

Fascia iliaca compartment block - Regional anesthesia

\section{Introduction}

Delirium or acute confusional state is defined as a disturbance of consciousness with inattention accompanied by a change in cognition or perceptual disturbance that develops over a short period (hours to days) and fluctuates over time [1]. Three subtypes of acute confusional state are recognized: hyperactive, hypoactive, and mixed. Hyperactive delirium is rare in the pure form and is associated with a better overall prognosis [2]. It is characterized by agitation, restlessness, attempting to remove catheters, and emotional lability [3]. Hypoactive delirium, which is very common and often more deleterious for the patient in the long term, remains unrecognized in 66-84\% of hospitalized patients, because hypoactivity on the part of a patient does not seem like a problem and may be missed [4-6]. This subtype is characterized by withdrawal, flat affect, apathy, lethargy, and decreased responsiveness [7].

Delirium is a common complication seen after hip fracture, affecting approximately $10-16 \%$ of patients [8]. It is associated with increased mortality at 1 year, delayed rehabilitation efforts, prolonged length of hospital stay, poorer functional outcomes, and increased risk of nursing home placement [9]. Although there are many hypothesized pathophysiologic mechanisms involved in the development of delirium, most are thought related to imbalances in neurotransmitters that modulate cognition, behavior, and $\operatorname{mood}[2,3]$. Predisposing factors to delirium include older age, diagnosis of dementia, depression, alcoholism, vision or hearing loss, history of mental status changes, mobility impairment, and dehydration $[4,6]$. Hospital-related or iatrogenic factors include physical restraints, malnutrition, more than three medications added, use of a bladder catheter, hypoxia, metabolic disturbances, electrolyte imbalances, withdrawal syndromes, acute infection (systemic and intracranial), seizures, hyperthermia, head trauma, vascular disorders, immobilization, sleep deficiency, psychiatric medications, and intracranial space-occupying lesions $[4,7]$.

Besides, hip fracture patients are at increased risk of delirium due to severe pain experienced upon arrival at the emergency department [10]. Findings from laboratory studies indicate that unmanaged pain, both acute and chronic, can affect mental status and might precipitate delirium, especially in elderly patients with hip fractures, who usually report a $50-70 \%$ incidence of severe pain in the first $24 \mathrm{~h}$ post injury $[11,12]$.
The aim of our study was to assess the effectiveness of fascia iliaca compartment block (FICB) for prevention of perioperative delirium in hip surgery patients who were at intermediate or high risk for this complication. The main hypothesis tested in this study was that FICB prophylaxis reduces delirium incidence in hip fracture patients.

\section{Materials and methods}

This was a prospective, randomized, placebo-controlled, clinical trial. A research team of orthopedic surgeons, neurologists, and nurses in a single 980-bed hospital, identified potentially eligible patients by systematically screening new admissions to one orthopedic ward, from July 2004 until March 2008. The study was approved by the local Ethics Committee and all patients signed informed consent. The study was performed in accordance with the ethical standards of the 1964 Declaration of Helsinki as revised in 2000.

Men and women aged 70 years and older admitted for hip fracture were considered for inclusion in the FICB prophylaxis study. Patients were excluded if they had delirium at admission, metastatic hip cancer, history of bupivacaine allergy, use of cholinesterase inhibitors, severe coagulopathy, Parkinsonism, epilepsy, levodopa treatment, delay of surgery of more than $72 \mathrm{~h}$ after admission, and inability to participate in interviews (profound dementia, respiratory isolation, intubation, aphasia, coma or terminal illness).

Initially, on admission, all included patients were divided into three groups, according to whether they were low, intermediate or high risk for perioperative delirium. Risk classification was based on presence of four predictive risk factors (severity of illness, measured using acute physiology age and chronic health examination, cognitive impairment, measured using the mini-mental state examination score, index of dehydration, measured using the ratio of blood urea nitrogen to creatinine, and visual impairment, measured using the standardized Snellen test) as described by Inouye et al. [13, 14]. Intermediate risk for postoperative delirium was defined as presence of one or two risk factors; high risk was defined as presence of three or more risk factors.

Eligible patients (those classified as being at intermediate or high risk for developing delirium) were sequentially randomly assigned to study treatment (FICB prophylaxis or placebo), according to a computer-generated randomization code. All participants were blinded to the treatment group. FICB was administered with a $0.25 \mathrm{mg}$ dose of $0.3 \mathrm{~mL} / \mathrm{kg}$ bupivacaine on admission and repeated daily every $24 \mathrm{~h}$ until delirium occurrence or hip surgery was performed. Twenty-four hours after hip surgery the same dose of FICB 
was re-administered and repeated daily every $24 \mathrm{~h}$ until delirium occurrence or discharge. A standardized FICB technique was used for the patients, as reported by Dalens et al. [15] without the need for a nerve stimulator, performed by orthopaedic surgeons. According to their technique, the puncture site is marked $1 \mathrm{~cm}$ caudal to the point dividing the lateral third and medial two-thirds of a line extended from the pubic tubercle to the anterior superior iliac spine. The skin is pierced with a sharp 24-gage needle, inserted perpendicular to the puncture site. Loss of resistance is noted first on passing the fascia lata and second on passing the fascia iliaca. After the second loss of resistance, a $0.25 \mathrm{mg}$ dose of $0.3 \mathrm{~mL} / \mathrm{kg}$ bupivacaine is injected slowly.

Block efficacy was tested by assessing sensory loss over the hip fracture area. An early predictor of optimal pain relief was sensory block of the inner thigh. Although continuous infusion of bupivacaine could be more convenient rather repeated blocks, complications such as overdose could occur. Placebo medication (water for injection) was identical in appearance to the active drug and was administered at the same site and in the same way as the FICB was injected. Intravenous and intramuscular analgesics were administered as needed in both groups. In these cases the patients received paracetamol intravenously (ampules for intravenous infusion of paracetamol $1 \mathrm{~g} / 6.7 \mathrm{ml}$ ) and intramuscularly pethidine $(50 \mathrm{mg})$ occasionally. Low-risk patients received no prophylactic medication.

Baseline screening and assessments were completed before hip surgery, within $12 \mathrm{~h}$ after admission, and included:

- Barthel index (range 0-20, lower scores indicating more functional dependency) [16]

- Chart review to determine APACHE II score (scale of $0-70$, with a cutoff score of 16 or higher indicating increased severity of clinical status) [17]

- Geriatric Depression Scale (15-item version self-rating scale for depression, range $0-15$, higher scores indicating depression) [18]

- Pre-existing cognitive impairment (mini-mental state examination (MMSE) score $\leq 24$ on a scale of $0-30$ ) [19]

- Ratio of blood urea nitrogen to creatinine

- Standardized Snellen test for visual impairment [20]

The primary outcome was perioperative delirium. Diagnosis of the syndrome was defined using the Diagnostic and Statistical Manual of Mental Disorders, 4th edition (DSM-IV) and Confusion Assessment Method (CAM) criteria [1, 21].

Secondary outcome variables were severity of delirium, delirium duration, and pain score. Delirium severity was measured using the Delirium Rating Scale, revised version-
98 [DRS-R-98, range 0 (no severity) to 45 (high severity)] [22]. Daily patient assessments using the MMSE, DRS-R98, and Digit Span test [assessment of attention, range 0 (no attention) to 42 (good attention)] were used to enable the DSM-IV and CAM diagnoses and assess delirium severity [23]. CAM and DRS-R-98 assessments were continued once delirium was diagnosed. This prospective study involved the use of a visual analogue scale (VAS) to record pain severity among patients.

All patients were assessed daily for efficacy and safety evaluations. Also, experienced nurses and geriatricians provided proactive geriatric consultation to all patients. The consultation was based on a structured multimodular protocol (geriatric medical attention, enhancement of orientation and cognition, sensory and mobility-improving advice, attention to pain and sleeping problems, extra attention to fluid and food intake, and patient, family, and nursing staff education) in order to minimized the chances for delirium developing [24]. All patients were operated under the same epidural anesthesia ( $15 \mathrm{ml} 0.5 \%$ bupivacaine). Subcapital and trochanteric hip fractures were treated with hemiarthroplasty and intramedullary nailing, respectively.

If perioperative delirium occurred, patients were treated according to standard procedures (haloperidol three times per day, lorazepam three times per day, or both in increasing doses, depending on symptoms of delirium) and assessed for delirium severity and duration $[4,5]$. Pain at time of delirium occurrence was treated with pethidine (50 mg intramuscularly).

Statistical calculations were performed using SPSS version 11 (SPSS, Inc., Chicago, IL) for Windows.

For the primary analysis of the intervention, delirium was considered a binary outcome (absent or present) according to its earliest occurrence. The results are expressed as relative risks with $95 \%$ confidence intervals (CIs) for the FICB group relative to the placebo group, with a relative risk less than 1.0 indicating a beneficial effect. Statistical significance was set at $P<0.05$.

\section{Results}

Of 324 patients initially admitted to our orthopaedic department, 287 entered the baseline phase. Thirty-seven patients were excluded; the reasons for not entering this phase were: 13 refused to participate, 11 were taking antipsychotic drugs, 4 were known to have Parkinsonism, 4 presented with pathologic hip fracture due to metastasis, 2 sustained acute myocardial infarction at admission, 2 were delirious at admission, and 1 presented with an old hip fracture. Of the 287 entering the baseline phase, 13 refused treatment with the study drug and 2 patients died before 
study treatment started. Fifty-three of 287 patients were not randomized because all four risk factors were absent (low risk). The remaining 219 eligible patients were randomized. Totally 12 patients were further excluded from both groups for different reasons: six lost to follow-up, three refused further participation, and three died (two pulmonary embolism and one stroke) between second and fourth day of admission. A flow diagram of the study is presented in Fig. 1.

Main features at the time of admission of the 102 patients randomized to FICB prophylaxis group and the 105 patients in the placebo group did not differ significantly (Table 1). The mean number of risk factors per patient was similar in the two groups. Also the low APACHE II scores and the high Barthel index scores indicated good clinical condition for both groups.

Delirium occurred in 33 (15.94\%) out of 207 patients randomized to the FICB prophylaxis and placebo groups.
The incidence of delirium in the FICB prophylaxis group was $10.78 \%$ (11/102), significantly different from the incidence $(23.8 \%, 25 / 105)$ in the placebo group (relative risk $0.45,95 \%$ CI $0.23-0.87$ ). The baseline features of patients in the FICB prophylaxis and placebo groups who developed delirium on follow-up did not differ significantly (Table 2).

Nine of 17 patients with high risk for delirium and included in the FICB prophylaxis group developed delirium, whereas 10 of 16 high-risk patients included in the placebo group became delirious (relative risk 0.84, CI $0.47-1.52$ ). Two of 85 patients with intermediate risk for delirium and included in the FICB prophylaxis group developed delirium, whereas 15 of 89 intermediate-risk patients included in the placebo group became delirious (relative risk 0.13, CI 0.03-0.53).

Although more ampules of paracetamol for intravenous infusion and pethidine for intramuscular infusion were
Fig. 1 Flow diagram of patients enrolled in the study

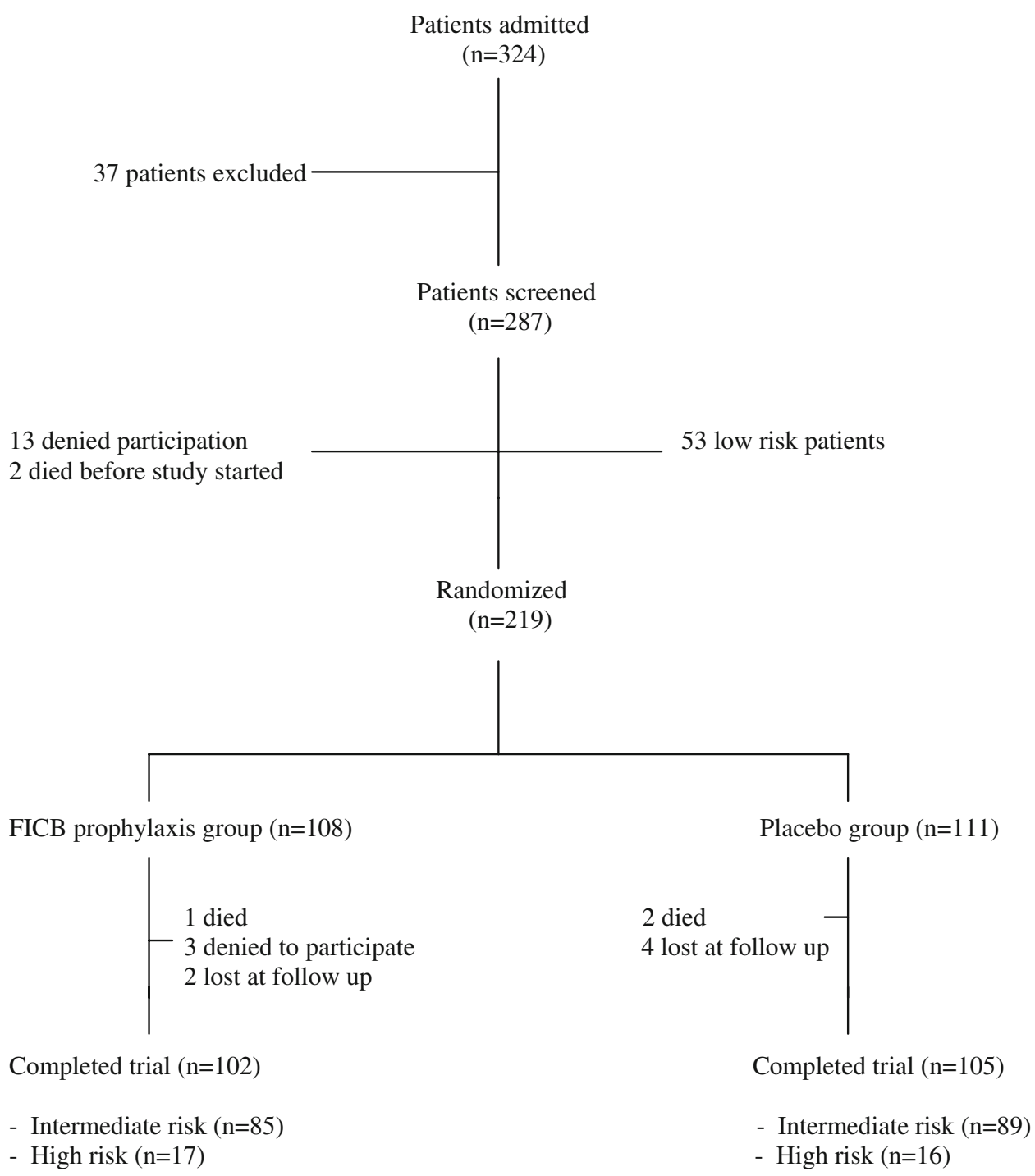


Table 1 Main features of 207 patients randomized to FICB prophylaxis and placebo group

\begin{tabular}{llll}
\hline & $\begin{array}{l}\text { FICB group } \\
(n=102)\end{array}$ & $\begin{array}{l}\text { Placebo group } \\
(n=105)\end{array}$ & $P$-value \\
\hline Age (years) & $72.3 \pm 4.1$ & $73.1 \pm 3.8$ & 0.28 \\
Female/male & $78 / 24$ & $76 / 29$ & 0.54 \\
Intertrochanteric/subcapital hip fracture & $72 / 30$ & $76 / 29$ & 0.58 \\
Barthel index & $18.45 \pm 1.32$ & $18.52 \pm 1.43$ & 0.72 \\
Geriatric Depression Scale & $1.22 \pm 1.04$ & $1.18 \pm 1.21$ & 0.53 \\
APACHE II score & $12.89 \pm 2.13$ & $12.97 \pm 1.82$ & 0.56 \\
MMSE score & $24.1 \pm 3.6$ & $24.43 \pm 3.2$ & 0.83 \\
Visual acuity & $0.4 \pm 0.12$ & $0.42 \pm 0.08$ & 0.68 \\
Dehydration index & $20.15 \pm 3.47$ & $20.24 \pm 3.15$ & 0.49 \\
Intermediate/high risk & $85 / 17$ & $89 / 16$ & 0.62 \\
\hline
\end{tabular}

\begin{tabular}{lllc}
\hline & $\begin{array}{l}\text { FICB group } \\
(n=11)\end{array}$ & $\begin{array}{l}\text { Placebo group } \\
(n=25)\end{array}$ & $P$-value \\
\hline Age (years) & $81.4 \pm 3.2$ & $81.8 \pm 2.9$ & 0.72 \\
Female/male & $9 / 2$ & $22 / 3$ & 0.32 \\
Intertrochanteric/subcapital hip fracture & $7 / 4$ & $18 / 7$ & 0.48 \\
Barthel index & $18.87 \pm 3.3$ & $18.56 \pm 4.2$ & 0.73 \\
Geriatric Depression Scale & $1.2 \pm 0.8$ & $1.3 \pm 0.62$ & 0.52 \\
APACHE II score & $15 \pm 2.8$ & $15.4 \pm 3.7$ & 0.36 \\
MMSE score & $21.34 \pm 3.2$ & $21.12 \pm 2.7$ & 0.32 \\
Visual acuity & $0.30 \pm 0.08$ & $0.34 \pm 0.01$ & 0.06 \\
Dehydration index & $20.34 \pm 5.1$ & $20.96 \pm 5.3$ & 0.48 \\
\hline
\end{tabular}

Table 2 Features of patients who developed delirium
There were no complications of FICB administration, except three local hematomas developed at the injection site which resolved spontaneously.

average 2.3 ampules of paracetamol/patient/day and 1.2 ampules of pethidine/patient/day and in the placebo group versus 0.65 ampules of paracetamol/patient/day and 0.48 ampules of pethidine/patient/day in the FICB prophylaxis group), no correlation between the number of ampules of paracetamol or pethidine needed and delirium occurrence was evidenced $(r=0.28, P=0.62)$. According to the VAS the pain scores in the two groups did not differ significantly (mean preoperative pain score of placebo group versus FICB group were 68.2 and 61.4, respectively, $P=0.59$; mean postoperative pain score of placebo group versus FICB group were 72.6 and 64.6, respectively, $P=0.34)$.

Severity of delirium according to the highest value of the DRSR-98 during an episode of delirium in patients from the FICB prophylaxis group was on average $14.34 \pm 3.6$, versus $18.61 \pm 3.4$ in the placebo group (mean difference 4.27, 95\% CI 1.8-5.64, $P<0.001$ ).

Mean duration of delirium in the FICB prophylaxis group was significantly shorter than in the placebo group (FICB $5.22 \pm 4.28$ days versus placebo $10.97 \pm 7.16$ days, $95 \% \mathrm{CI}=3.87-7.62, P<0.001)$.

\section{Discussion}

Fascia iliaca compartment block is an anterior thigh regional anesthetic block of lumbar plexus. As it is injected into the fascia iliaca compartment, it spreads to nerves innervating the hip and could reach the femoral and the lateral cutaneous nerve of the thigh [15]. It then spreads medially and proximally into a space between the psoas and iliacus muscles, which act as a barrier to the substance, reaching the obturator nerve in almost one-third of cases [25].

Fascia iliaca compartment block technique is remarkably simple to learn and use. It is also considered to be safe. The site of injection with the FICB is distant from any nerves or blood vessels, which theoretically should eliminate the possibility of intravascular or intraneural injection [25]. However it is associated with rapid onset of pain relief. Foss et al. [26] supported the use of FICB in acute 
management of hip fracture pain because it is an effective procedure. Forty-eight patients with hip fracture received FICB injection in their study. They reported that FICB provides excellent pain relief both at rest and on $15^{\circ}$ leg lift in hip fracture patients. Candal-Couto et al. [27] studied 30 patients with hip fracture who received FICB. They concluded that this type of regional anesthesia can provide significant benefit in the preoperative period and allow patients to sit up more comfortably while they await surgery. Monzo et al. [25] reported 63 patients who sustained hip fracture and were administered a single dose of FICB. They concluded that FICB injection produced significant benefit by controlling pain, far better than standard parenteral medications, which lasted $8 \mathrm{~h}$ at least. Pain was diminished dramatically in the first $15 \mathrm{~min}$ after the injection. Also they mentioned that analgesic requirements after FICB injection were minimal. They also first suggested that FICB analgesic effect could be beneficial for preventing delirium after hip fracture.

In fact the relationship between pain and delirium has been studied in patients recovering from hip fracture. There is evidence from carefully controlled laboratory studies that pain can impact on cognition and thus might precipitate delirium, particularly in hip fracture patients. Morrison et al. [28], in a large prospective study of 541 hip fractures, reported that $16 \%$ of patients became delirious and that severe pain was associated with a ninefold risk of developing delirium. Several studies suggest that, when pain is managed in hip fracture patients, delirium is reduced. Marcantonio et al. [11], in a randomized study of hip fracture patients undergoing surgery, reported that nine specific interventions, with pain management also included, statistically reduced delirium by one-third. Pain management in this study consisted of round-the-clock acetaminophen ( $1 \mathrm{~g}$, four times daily), low-dose subcutaneous morphine early in the postoperative period, and oxycodone later in the postoperative period. In another study of hip fracture patients, a nurseled interdisciplinary intervention program for delirium was implemented in the experimental group using a shortacting weak opioid analgesic in combination with acetaminophen, while the control group received usual care. Although the incidence of delirium occurrence was the same in both groups, the patients in the experimental group experienced much shorter duration of delirium, with less severity [12].

Since Monzo et al. reported their belief that FICB prophylaxis could prevent delirium occurrence, no trial has studied the effect of FICB prophylaxis in preventing delirium in hip fracture patients. Herein, this effect of FICB prophylaxis is studied. According to our findings, there was significant difference in the incidence of delirium between the FICB prophylaxis and placebo groups. However no significant difference was observed among high-risk patients between the FICB prophylaxis and placebo groups in terms of delirium incidence. On the other hand FICB prophylaxis significantly prevented delirium occurrence in intermediate-risk patients. Thus FICB prophylaxis could be beneficial, particularly for intermediate-risk patients. Additionally, severity and duration of delirium were significantly less in the intervention group. These findings strongly suggest that FICB prophylaxis may lead to smaller numbers of hip fracture patients developing delirium.

Moreover, FICB prophylaxis in our study was well tolerated, and its low cost enhances the chances that this intervention will be applied. Although patient controlled analgesia (PCA) pumps and oral analgesics are alternative treatments, they are associated with obvious disadvantages. Crombez et al. [29] supported that, if a patient is in severe pain, there will be cognitive interference and the patient may not be able to complete the task of pushing the button on the PCA pump. In these cases pain may get out of control [30]. Also the beneficial effect of FICB versus oral analgesics has been evidenced [27].

This study has several limitations. Although we controlled for perioperative risk factors for delirium, we did not examine the impact of drugs other than paracetamol and pethidine.

In conclusion, delirium incidence was reduced after FICB injection in patients who had sustained hip fracture, especially those who were at intermediate risk for this complication. FICB, either in its own right or versus opioid regimens, leads to better delirium outcomes.

Conflict of interest statement The authors declare that they have no conflict of interest related to the publication of this manuscript.

\section{References}

1. American Psychiatric Association (2000) Diagnostic and statistical manual of mental disorders, 4th edn, text revision. American Psychiatric Association, Washington, DC

2. Meagher DJ, Trzepacz PT (2000) Motoric subtypes of delirium. Semin Clin Neuropsychiatry 5:75-85

3. O'Keeffe ST, Lavan JN (1999) Clinical significance of delirium subtypes in older people. Age Ageing 28:115-119

4. Inouye SK (1994) The dilemma of delirium: clinical and research controversies regarding diagnosis and evaluation of delirium in hospitalized elderly medical patients. Am J Med 97:278-288

5. Sanders AB (2002) Missed delirium in older emergency department patients: a quality-of-care problem. Ann Emerg Med 39:338-341

6. Hustey FM, Meldon SW (2002) The prevalence and documentation of impaired mental status in elderly emergency department patients. Ann Emerg Med 39:248-253

7. Meagher DJ, Hanlon DO, Mahony EO et al (2000) Relationship between symptoms and motoric subtype of delirium. J Neuropsychiatry Clin Neurosci 12:51-56

8. Morrison RS, Magaziner J, Gilbert M et al (2003) Relationship between pain and opioid analgesics on the development of 
delirium following hip fracture. J Gerontol A Biol Sci Med Sci 58:76-81

9. Pompei P, Foreman M, Rudberg M, Inouye S, Braund V, Cassel C (1994) Delirium in hospitalized older persons: outcomes and predictors. J Am Geriatr Soc 42:809-815

10. Roberts HC, Eastwood H (1994) Pain and its control in patients with fractures of the femoral neck while awaiting surgery. Injury 25:237-239

11. Marcantonio ER, Flacker JM, Wright RJ, Resnick NM (2001) Reducing delirium after hip fracture: a randomized trial. J Am Geriatr Soc 49:516-522

12. Milisen K, Foreman MD, Abraham IL, De Geest S, Godderis J, Vandermeulen E, Fischler B, Delooz HH, Spiessens B, Broos PL (2001) A nurse-led interdisciplinary intervention program for delirium in elderly hipfracture patients. J Am Geriatr Soc 49:523532

13. Inouye SK, Viscoli CM, Horwitz RI et al (1993) A predictive model for delirium in hospitalized elderly medical patients based on admission characteristics. Ann Intern Med 119:474-481

14. Inouye SK, Charpentier PA (1996) Precipitating factors for delirium in hospitalized elderly persons. Predictive model and interrelationship with baseline vulnerability. JAMA 275:852-857

15. Dalens B, Vanneuville G, Tanguy A (1989) Comparison of the fascia iliaca block with the 3 -in-1 block in children. Anesth Analg 69:705-713

16. Mahoney FI, Barthel DW (1965) Functional evaluation: the Barthel index. Md State Med J 14:61-65

17. Knaus WA, Draper EA, Wagner DP et al (1985) APACHE II. A severity of disease classification system. Crit Care Med $13: 818-829$

18. Yesavage JA, Brink TL, Rose TL et al (1982) Development and validation of a geriatric depression screening scale: a preliminary report. J Psychiatr Res 17:37-49

19. Folstein MF, Folstein SE, McHugh PR (1975) Mini-mental state. A practical method for grading the cognitive state of patients for the clinician. J Psychiatr Res 12:189-198
20. Hetherington R (1954) The Snellen chart as a test of visual acuity. Psychol Forsch 24:349-357

21. Inouye SK, van Dyck CH, Alessi CA et al (1990) Clarifying confusion: the confusion assessment method. A new method for detection of delirium. Ann Intern Med 113:941-948

22. Trzepacz PT, Baker RW, Greenhouse J (1988) A symptom rating scale for delirium. Psychiatry Res 23:89-97

23. Cummings JL (1985) Clinical neuropsychiatry. Grune \& Stratton, Orlando

24. Marcantonio ER, Goldman L, Mangione CM et al (1994) A clinical prediction rule for delirium after elective noncardiac surgery. JAMA 27:134-139

25. Monzon D, Iserson K, Vazquez J (2007) Single fascia iliaca compartment block for post-hip fracture pain relief. J Emerg Med 32(3):257-262

26. Foss N, Kristensen B, Bundgaard M, Bak M, Heiring C, Virkelyst C, Hougaard S, Kehlet H (2007) Fascia iliaca compartment blockade for acute pain control in hip fracture patients. A randomized, placebo-controlled trial. Anesthesiology 106:773-778

27. Candal-Couto JJ, McVie JL, Haslamb N, Innesa AR, Rushmerb J (2005) Pre-operative analgesia for patients with femoral neck fractures using a modified fascia iliaca block technique. Injury 36:505-510

28. Morrison RS, Magaziner J, Gilbert M, Koval KJ, McLaughlin MA, Orosz G, Strauss E, Siu AL (2003) Relationship between pain and opioid analgesics on the development of delirium following hip fracture. J Gerontol Med Sci 58A:76-81

29. Crombez G, Eccleston C, Baeyens F, Eelen P (1997) Habituation and the interference of pain with task performance. Pain 70:149_ 154

30. Keita H, Geachan N, Dahmani S, Couderc E, Armand C, Quazza M, Mantz J, Desmonts JM (2003) Comparison between patientcontrolled analgesia and subcutaneous morphine in elderly patients after total hip replacement. Br J Anaesth 90(1):53-57 